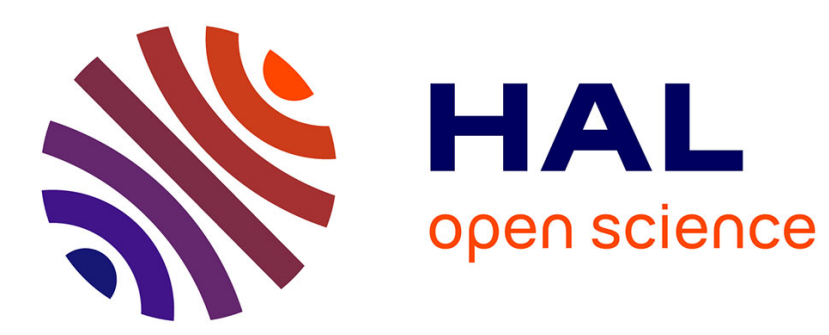

\title{
A genetic algorithm with decimal coding for the estimation of kinetic and energetic parameters
}

Laurent Balland, Lionel Estel, Jean-Marie Cosmao, Nordine Mouhab

\section{To cite this version:}

Laurent Balland, Lionel Estel, Jean-Marie Cosmao, Nordine Mouhab. A genetic algorithm with decimal coding for the estimation of kinetic and energetic parameters. Chemometrics and Intelligent Laboratory Systems, 2000, 50 (1), pp.121-135. 10.1016/S0169-7439(99)00057-X . hal-02138371

\section{HAL Id: hal-02138371 \\ https://hal.science/hal-02138371}

Submitted on 13 Dec 2019

HAL is a multi-disciplinary open access archive for the deposit and dissemination of scientific research documents, whether they are published or not. The documents may come from teaching and research institutions in France or abroad, or from public or private research centers.
L'archive ouverte pluridisciplinaire HAL, est destinée au dépôt et à la diffusion de documents scientifiques de niveau recherche, publiés ou non, émanant des établissements d'enseignement et de recherche français ou étrangers, des laboratoires publics ou privés. 


\title{
A genetic algorithm with decimal coding for the estimation of kinetic and energetic parameters
}

\author{
L. Balland *, L. Estel, J.-M. Cosmao, N. Mouhab \\ LRCP, INSA Rouen, Place E. Blondel, B.P. 08, F-76131 Mont Saint Aignan Cedex, France
}

Received 15 July 1999; accepted 21 September 1999

\begin{abstract}
A hybrid estimation method is proposed to estimate simultaneously the kinetic and energetic parameters of chemical models. This method combines a genetic algorithm with a local convergence method, the former generating initialisation points for the latter. The method is applied to a real complex chemical system: the saponification of ethyl acetate at a concentration of $1 \mathrm{~mol} \mathrm{l}^{-1}$. The reaction is carried out in a calorimetric reactor which determines experimental profiles of power delivered or absorbed by the reaction mass. A model, based on mass and energy balances in the reaction mass, including the kinetic and energetic parameters of the reaction, allows to calculate the power profile. The comparison between the experimental and calculated profiles gives the criterion to minimise. The parameters of the model are successfully determined. The hybrid method determines more efficiently and more rapidly the solution of the problem than a set of several estimations with a local convergence method. (c) 2000 Elsevier Science B.V. All rights reserved.
\end{abstract}

Keywords: Genetic algorithm; Kinetic parameter estimation; Saponification of ethyl acetate; Calorimetric reactor

\section{Introduction}

The knowledge of the thermal and kinetic behaviour of the chemical reaction mass in an industrial process is essential, both during the conception phase and in safety studies. The modelling of chemical processes needs the estimation of non measurable parameters, such as kinetic constants. The more complex chemical systems are, the more sophisticated parameter estimation tools are required.

The estimation method of kinetic and energetic parameters described here consists in carrying out the

\footnotetext{
* Corresponding author. Tel.: + 33-235-5284-56; fax: + 33235-5284-52; e-mail: laurent.balland@insa-rouen.fr
}

chemical reaction in a calorimetric reactor. The profile of power delivered or absorbed by the reaction mass is measured against the time. Taking the same experimental conditions, the power profile is calculated off line from a mathematical model, based on mass and energy balance. This model involves the kinetic and energetic parameters which need to be evaluated. For each reaction, the parameters are enthalpy, the kinetic constants as well as the reagent partial orders, and occasionally, for equilibria, the equilibrium constant. This profile calculated thanks to the model is compared to the experimental one. The difference between the two curves is assumed to be the criterion to be minimised, by the means of changing the kinetic and energetic parameters [1]. 
The number of parameters to be estimated increases very rapidly with the complexity of the physico-chemical model. It is necessary to use a robust estimation method, allowing the whole parameter space to be explored in order to determine the global solution to the problem, and not merely a local solution. The drawback with local convergence estimation methods is that they are only able to find the solution in the neighbourhood of the starting point. So if the problem presents several local minima, the solution found will be the one nearest the starting point. And a physical system only has one solution, which is the global minimum.

Genetic Algorithms (GAs) are estimation methods which are independent of the starting point and able to search in the entire space. They have been the subject of several studies, particularly in the field of chemical engineering. D.B. Hibbert [2] proposed a good overview of applications of GA in chemistry. One difficulty of these methods is that they require a large number of estimations of the criterion.

This paper presents a hybrid method, including a GA which is combined to a local convergence method. The GA is used to generate suitable initialisation points for local convergence methods. The GA uses decimal coding to carry out cross-over and mutation operations.

This method was used to estimate the energetic $(\Delta H)$ and kinetic (pre-exponential factor, activation energy, order) parameters of the reaction of ethyl acetate saponification in water-alcohol reaction mass [3].

\section{Description of the numerical method}

\subsection{General algorithm / principle}

The algorithm of the method is presented in Fig. 1. It includes two main steps, the first being the GA itself. The GA manages a population of individuals. Each individual corresponds to a point in the parameter space. This point is associated with a set of values for each parameter, which allows to calculate a criterion. The lower the criterion is, the better the individual is.

The GA step is used for detecting parameter space zones where the criterion is of greatest interest. The population of individuals is split in groups. The belonging of an individual to a group or another is decided according to the Euclidean distance between individuals.

If the number of groups $N b g r$ is too high, the GA is continued until Nbgr is low enough. The limit is fixed by the user. Then, the second main step is initiated. It consists in initialising a local convergence method with the best individual from each group.

The different steps of the GA are detailed in the following paragraphs.

\subsection{Choice of the first generation}

In the GA, the first step consists in creating the first generation of individuals. This can be done in different ways.

\subsubsection{Random distribution}

Points are chosen randomly in the whole research space. This is the most common solution as it is the most straightforward to implement. However, if the number of points is low, the points will not be very evenly scattered. Large zones will not be explored due to the concentration of points in other zones. To have a sufficient uniformity of distribution, it is necessary to increase the number of points, which will increase the length of calculation time.

\subsubsection{Regular lattice}

To avoid this drawback, a simple technique consists in building a regular lattice of the parameter space, which allows all the zones of space to be inspected. However, if one of the parameters has a short range, then the variations for this parameter will not greatly influence the value of the criterion.

A limit situation appears when some of the parameters are useless for the system: they do not influence the value of the criterion. It is calculated for several sets of values of useless parameters associated with unchanged values of the other parameters. This will, of course, give no new information for seeking the optimum of the criterion and will therefore be a waste of computation time.

For example, let us consider a kinetic model with three parameters to estimate, one of them being the 


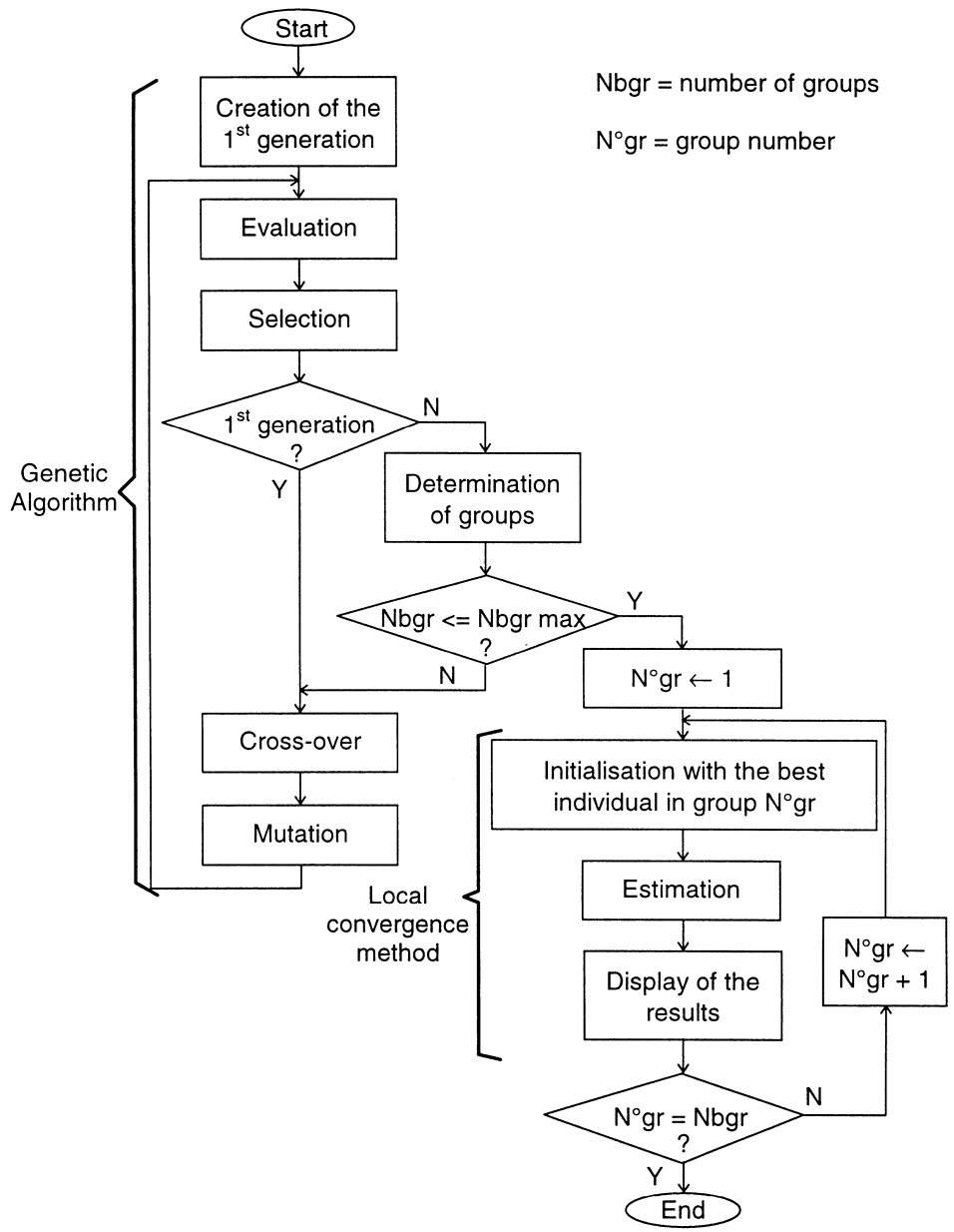

Fig. 1. Algorithm of the method.

enthalpy of reaction $\Delta H$. Thanks to the first principle of thermodynamics, its value can be estimated a priori by integrating the experimental power data, divided by the number of reacting moles.

$Q=-n_{0} \Delta H=\int q \mathrm{~d} t$.

It is then possible to define a very narrow range of variation for this parameter. If a lattice with 10 points per parameter is chosen for the three parameters, that makes 1000 points to calculate. However, only 100 points will have very different values of the criterion. The 900 other points will have approximately the same value of the criterion with one of the
100 first points because the only parameter which makes the difference is the enthalpy of reaction which does not have a great influence on the value of the criterion.

\subsubsection{Uniform lattice}

This drawback can be avoided thanks to a uniform lattice. In this lattice, it is necessary that, whatever the parameter, one value of this parameter be met for only one single point of the lattice. Sobol [4] proposed an algorithm to calculate the coordinates of the points to respect this condition. An example for the distribution of 100 points in two dimensions is given in Fig. 2. This figure shows the three kinds of distri- 


\section{Random Distribution}

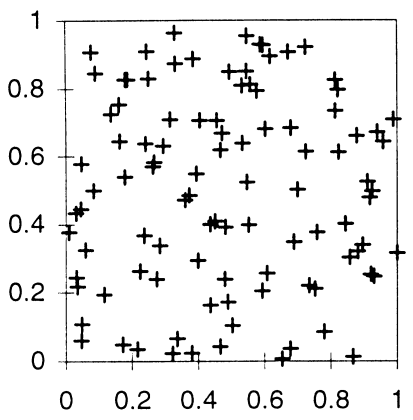

Distribution with Regular Lattice

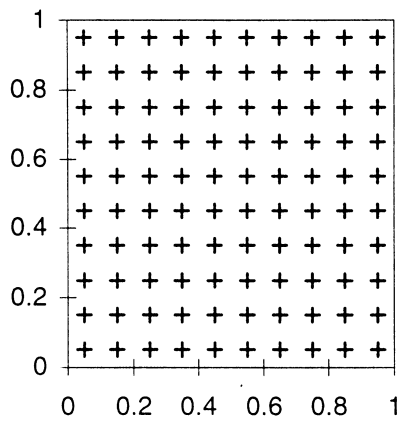

Distribution with Uniform Lattice

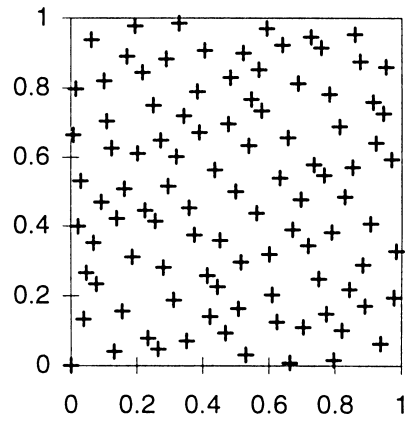

Fig. 2. Distribution of 100 points in two dimensions.

bution: random, regular lattice and uniform lattice (Sobol). The latter technique of initialisation has been used in our method.

\subsection{Evaluation}

The evaluation step consists in using the values of the parameters for each individual in the model under study. The model then calculates the power delivered by the reaction mass against the time. This profile is compared to the experimental one. The area between the two curves, which is an energy, is calculated and is used as the criterion of the individual. In most cases, in order to estimate all the parameters, it is necessary to carry out several experiments to take the different physical data into account: variations in temperature, in concentration... The criterion to be minimised is the sum for all experiments:

$f=\sum_{k=1}^{\mathrm{NE}} \Delta t_{k}\left(\sum_{\text {start }}^{\text {end }}\left|q_{\mathrm{R}, k}^{\exp }(t)-q_{\mathrm{R}, k}^{\text {calc }}(t)\right|\right)$

where NE is the number of experiments and $\Delta t_{k}$ is the sampling period of experiment $k$. The values of the parameters to estimate are supposed to remain constant for the operating conditions of all the experiments taken into account.

The information $\left(q_{\mathrm{R}, k}^{\exp }(t)\right)$ included in each experiment is proportional to the number of reacting moles. But if there are more reacting moles, the power re- leased will be greater, and the difference calculated, as it is the absolute difference and not the relative difference, will also be greater. If the reaction is fast, the power will be released on a short time, but with more power. On the contrary, if the reaction is slow, the number of points will be greater, as the time will be longer, but the power, and then the differences, will be smaller.

We would like to point out the fact that the signal measured is a power and not a concentration. This means that the information is a differential term, which is directly proportional to the rate of reaction. It implies that even if the power flattened out, with a significant value, there is still information to gather.

The criterion, calculated thanks to Eq. (2), corresponds to the adaptation of the individual to "survive" in the new generations. The lower the criterion is, the more likely the individual will be kept during the selection step.

\subsection{Selection}

For the population to evolve in the parameter space, it is necessary to eliminate some individuals and to create new ones, as well as to keep a constant population size. Some individuals are therefore selected to be used in the reproduction step during which the population is regenerated.

An elitist method was chosen: only a percentage of the best individual was kept. 


\subsection{Reproduction}

\subsubsection{Coding}

Regarding the reproduction step, a first solution was studied, without any coding as proposed by Bicking et al. [5]. The value of the parameters is kept as it is, and the children are chosen randomly in the subspace defined by two parents. This method did not give interesting results due to the natural tendency of this method to create children in the middle of the space, and then children are excluded from the border. That is why a method using coded parameters was preferred.

In most GAs, the coding of values of parameters is based on the binary representation of numbers. For the sake of simplicity, decimal coding was preferred.

All parameters are reduced and centred between 0 and 1 (cf. Section 2.7.2). This infers that their decimal value is written as: $0 . x y z \ldots$ with $x, y$, and $z$ decimal characters (included between ' 0 ' and ' 9 '). Number coding is done by converting their decimal value in an ASCII character chain, without the first 0 and the decimal point. Thus 0.14016 will be coded as: ' 1 ', ' 4 ', ' 0 ', ' 1 ', ' 6 '. It is with this chain of characters that the cross-over and mutation operations will be carried out.

The length of the chains will state the necessary precision. However, it is not possible to exceed the precision of the binary representation of the real numbers. As the computation code is written for real numbers in double precision, the coding chain is limited to a maximum of 15 characters.

\subsubsection{Cross-over}

Two parents are chosen randomly from among the individuals selected. The parents' chains are combined to create a new child.

The coding chains of the two parents are cut at certain points (the same for both parents) and the chain segments are interchanged between the two parents. Two new children are created. One of the two children is randomly chosen to complete the population.

\subsubsection{Mutation}

Each character in the coding chain has a probability $m$ of changing randomly. The value of $m$ is low (few percentage) and decreases linearly with the number of generations, as proposed by Hibbert [6]. Indeed, during the first generations, the individuals are far from the solution and it is necessary to make them evolve. On the contrary, when the number of generations is higher, then the individuals are nearer from the solution and it is no longer necessary to make them evolve so much.

\subsection{Determination of groups}

The calculation of the criterion of each individual (Eq. (2)) is quite long. There are a lot of points per experiment (there could be several thousands), there could be several experiments (up to 10 or more). For complicated cases, the length of calculation time will exceed several seconds, even on fast computer. So there is a need in a fast converging method. The idea was not to let the GA converge but detect before the formation of local minima. Then a local convergence method is much faster than GA.

To bring to the fore the creation of aggregates at the end of a generation, the individuals selected are classified by rising order of the criterion. The Euclidean distance from each individual to the best of them is calculated; if it is less than the distance predefined by the user, this individual belongs to the first group, otherwise it is the first element of the second group; and so on. Each group is characterised by its best point and its number of points. As the process goes on, there is a progressive decrease in the number of groups. According to the number of groups, the user can decide either to create a new generation or to continue with the second main step: the local convergence method. The software also serves to define a minimum number of groups from which the second main step of computation is started automatically. This allows to compute without any intervention by the user. Otherwise, intervention is required at the end of each generation.

As said before, the second main step of computation is to start a criterion minimisation method from the best individual of each group. The method chosen is Rosenbrock's method with constraints [7]. It then gives as many solutions as there are groups. Different situations can then occur: either a global optimum clearly appears, or several optima give similar values of the criterion and the choice among them 
is made by taking their physical meanings into account.

\subsection{Choice of parameters}

To increase the efficiency of the parameter estimation method, it is important to choose the most sensitive parameters possible to estimate. Bearing this in mind, some variables may be changed.

\subsubsection{Kinetic constants}

Among the parameters to estimate, the kinetic constants are often difficult to estimate. These constants $k_{i}$ follow an Arrhenius' law (with pre-exponential factor $A_{i}$ and activation energy $E_{i}$ ):

$k_{i}=A_{i} \exp \left(\frac{-E_{i}}{R T_{\mathrm{r}}}\right)$.

Several authors (Chen [8], Billardello [9]) showed the advantage of not using $A_{i}$ and $E_{i}$, as parameters to identify, but a function of these. Indeed, the criterion function should be equi-sensitive to all the parameters in order to obtain an efficient minimisation method: partial derivatives of the criterion function over each parameter must have approximately the same value. This is why, as proposed by Chen [8], it is better to estimate not $A_{i}$ directly, but its logarithm: $\ln A_{i}$.

If it is necessary to estimate simultaneously preexponential factor $A_{i}$ and activation energy $E_{i}$, it is then possible to use crossed reparameterisation. Pritchard and Bacon [10] proposed:

$\varphi_{i}=\ln A_{i}-E_{i} / R T^{*}$

$\psi_{i}=\ln E_{i} / R$

where $T^{*}$ is a reference temperature. The parameters $A_{i}$ and $E_{i}$ become $\varphi_{i}$ and $\psi_{i}$. Swaels [11] notes that $\varphi_{i}$ and $\psi_{i}$ have no physical meanings, which makes their limits and initial values difficult to choose a priori. Moreover, the exploration of the domain presents a discontinuity for $E_{i}=R$. This is why Swaels prefers to use the following reparameterisation:

$k_{i, 1}=A_{i} \exp \left(-\frac{E_{i}}{R T_{1}}\right)$
$k_{i, 2}=A_{i} \exp \left(-\frac{E_{i}}{R T_{2}}\right)$ with $T_{1}$ and $T_{2}$ as two reference temperatures, taken, most of the time, at the extremes of the temperature range corresponding to the experimental data. The values of $A_{i}$ and $E_{i}$ are then given by the following equations:

$A_{i}=\frac{k_{i, 1}}{\exp \left(-\frac{E_{i}}{R T_{1}}\right)}$

$E_{i}=\left(\ln k_{i, 1}-\ln k_{i, 2}\right) \frac{R T_{1} T_{2}}{T_{1}-T_{2}}$.

The method now consists in identifying $k_{i, 1}$ and $k_{i, 2}$, which are the values of the kinetic constant for two temperatures. To improve the distribution of values, it is possible to carry out the estimation with the logarithm of the kinetic constants.

To show the interest of changing parameters, the response surface of the criterion function was represented, either as a function of $E$ and the logarithm of $A$, or as a function of the kinetic constant at two different temperatures. A simple real case was chosen, with only one reaction, the enthalpy of which was already known. Figs. 3 and 4 show the difference of profile according to the parameters used. In Fig. 3, which represents the response surface with the logarithm of $A$ and the activation energy $E$, the surface presents a deep crevasse, the bottom of which is slightly curved in the neighbourhood of the optimum. Conversely, in Fig. 4, which represents the same function but with changed parameters, the surface presents only a well and the optimum of the function is therefore much easier to find. The forbidden zone corresponds to negative values of the activation energy. It is clear from these figures that changing parameters allows the function to be much more sensitive to their values, which will thus be easier to estimate.

Pigram et al. [12] have proposed another changing in parameters. They determine the equation of the line in the valley described in Fig. 3, and recast the expression of the parameters to ensure the parameters be searched in a narrow band in the bottom of the valley. This method was not suitable for us as we have no way to determine the valley in advance. 


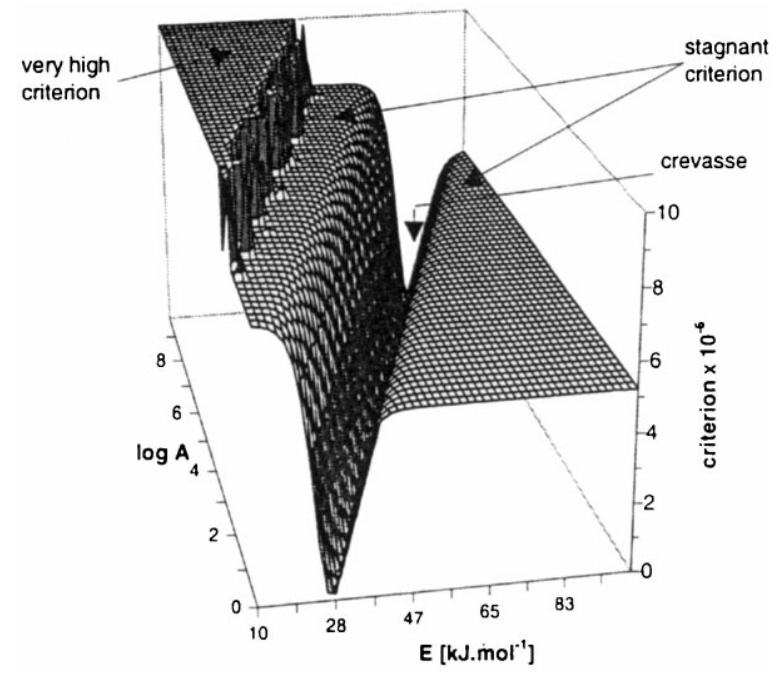

Fig. 3. Response surface of the criterion function without change of parameters.

\subsubsection{Normalisation}

All the parameters are systematically centred and reduced to between 0 and 1 . This technique is very useful for the GA.

At the end of each generation, the program computes the Euclidean distance between the points in the parameter space. The ranges of variation in the values of the parameters have to be the same for all pa- rameters. If all parameters vary between 0 and 1 , then the direction does not affect the distance.

\subsection{Validation}

The method was validated using numerical examples from the literature, such as Griewangk's function [13] or Schwefel's function [14]. These functions

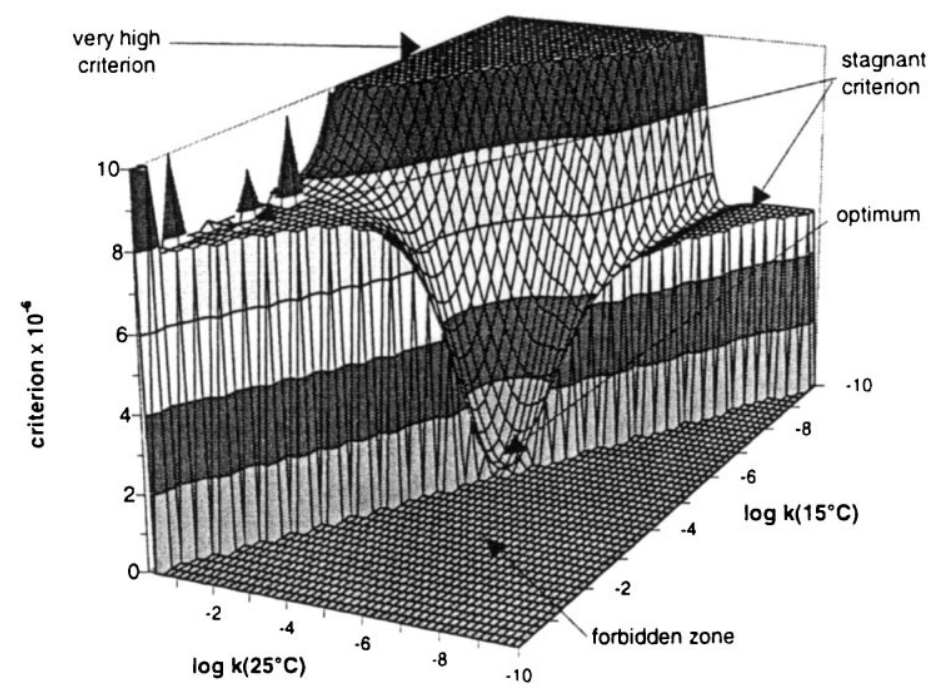

Fig. 4. Response surface of the criterion function with change of parameters. 
are known to be strongly multimodal. The estimation method was able not only to determine the best minimum, but also some of the secondary minima.

\section{Application}

\subsection{Chemical model studied}

The reaction under study is the saponification of ethyl acetate (A), by sodium hydroxide (B) to give sodium acetic acid salt (C) and ethanol (D).

$\mathrm{AcOEt}+\mathrm{Na}^{+} \mathrm{OH}^{-} \rightarrow \mathrm{AcO}^{-} \mathrm{Na}^{+}+\mathrm{EtOH}$

$\mathrm{A}+\mathrm{B} \rightarrow \mathrm{C}+\mathrm{D}$

The reaction is carried out in concentrated $(1 \mathrm{~mol}$ $1^{-1}$ ) hydroalcoholic mixture, in a range of temperature between $15^{\circ} \mathrm{C}$ and $30^{\circ} \mathrm{C}$.

To avoid equilibrium conditions, between acidalcohol and ester-water, the sodium hydroxide is in large excess (1.5 times more than ester), and is in the reactor at the beginning of the reaction. With such concentration, the ester is not rapidly soluble in water. Moreover, there is a difference in the densities between water and ester. That is the reason why a hydroalcoholic mixture is preferred: $50 \%$ in mass for ethanol, $4.5 \%$ for sodium hydroxide and $45.5 \%$ for water.

The Mettler ${ }^{\circledR}$ RC1 glass made calorimetric reactor presented in Fig. 5 works at atmospheric pressure. It is composed of a jacketed reactor with tangential input for the heat transfer fluid. The capacity of the reactor is 21 . It is equipped with a Pt100 temperature probe, an electrical calibration heating, a stirring system (either anchor or turbine with four baffles) and an introduction system (two dosing pumps and two balances, with measurement of the temperature at introduction). The heating-cooling system, which uses a single heat transfer fluid, works within a temperature range from $-15^{\circ} \mathrm{C}$ to $+200^{\circ} \mathrm{C}$.

An exploitation software solves the energetic balance on the reaction mass every $2 \mathrm{~s}$, thanks to Eq. (10) which enables the power released or absorbed $\left(q_{\mathrm{R}}^{\exp }\right)$ by the physical and chemical transformations of the reaction mass to be calculated. This balance is established with the hypotheses that the agitation is per-

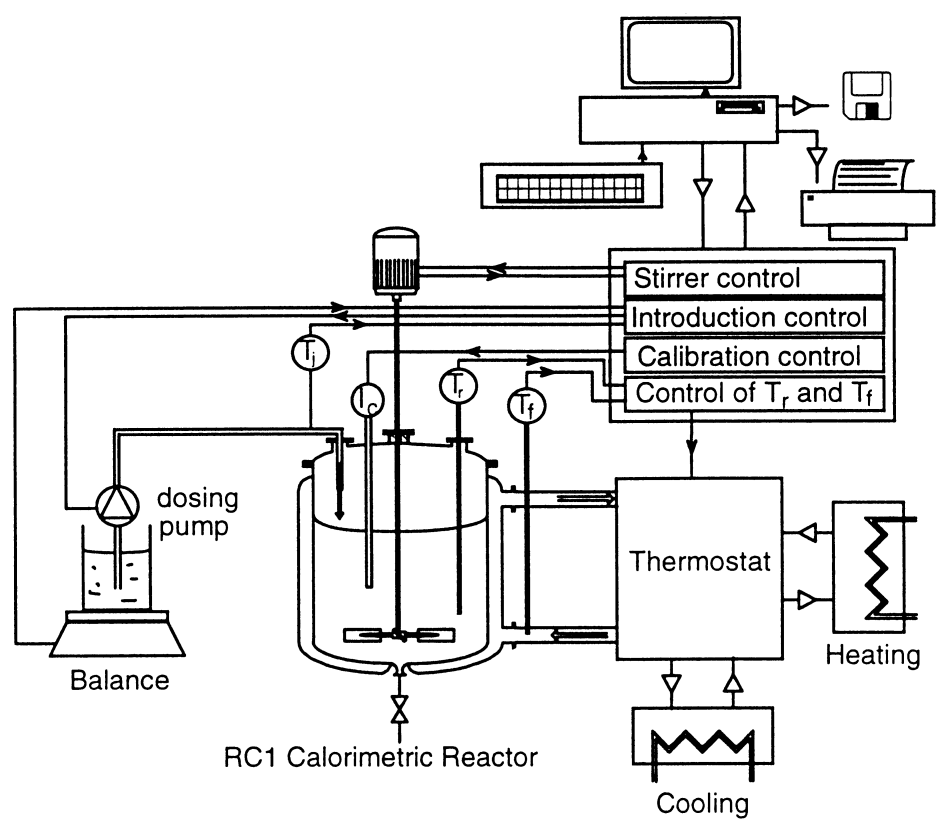

Fig. 5. Diagram of the RC1 Calorimetric Reactor. 
fect and that the temperature is homogeneous throughout the jacket (Fig. 6):

$$
\begin{array}{r}
q_{\mathrm{R}}^{\exp }+\sum(\mathrm{m} . \mathrm{cp}) \frac{\mathrm{d} T_{\mathrm{r}}}{\mathrm{d} t}+\mathrm{U} \cdot \mathrm{A} .\left(T_{\mathrm{r}}-T_{\mathrm{f}}\right) \\
+\sum_{j=1}^{\mathrm{NS}} \dot{n}_{j}^{\mathrm{E}} C p_{j}\left(T_{\mathrm{r}}-T_{j}\right)+q_{\mathrm{loss}}=0 .
\end{array}
$$

During each experiment, which are carried out at different temperatures, the calorimetric reactor is filled with 1.51 of the hydroalcoholic mixture. First of all, calibrations are carried out to calculate the global heat transfer conductance (U.A) and the heat capacity of the reaction mass ( $\left.\sum \mathrm{m} . c p\right)$. These values are required to calculate the experimental profile of the power released or absorbed by the reaction mass with Eq. (10). Then $88 \mathrm{~g}(1 \mathrm{~mol})$ of ethyl acetate is introduced in $85 \mathrm{~s}$. The reaction is supposed to be finished when no more power is measured from the reaction mass. Finally, new calibrations are carried out to take into account the evolution of the heat conductance and the heat capacity due to the reaction. The measurements allow to calculate the experimental power profile released or absorbed by the reaction mass $q_{\mathrm{R}}^{\mathrm{exp}}$, as explained by Balland et al. [15].

To determine the parameters in the whole range of temperature, three experiments are carried out: the first one with a variation of temperature between $15^{\circ} \mathrm{C}$ and $30^{\circ} \mathrm{C}$ during the same time as the introduction of ester; the second one by trying to maintain the temperature at $15^{\circ} \mathrm{C}$; and the third one by trying to maintain it at $30^{\circ} \mathrm{C}$.

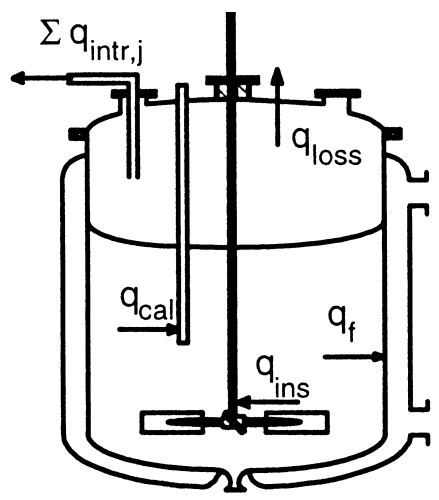

Fig. 6. Powers exchanged with the reaction mass.
The profile of power measured by the calorimetric reactor shows a small negative peak at the beginning of the introduction. This is due to an excess heat. To take such a heat into account, a reaction for mixing $\{1\}$ is introduced in the stoichiometric model:

$$
\begin{aligned}
& \mathrm{A}^{\text {pure }} \rightarrow \mathrm{A} \\
& \mathrm{A}+\mathrm{B} \rightarrow \mathrm{C}+\mathrm{D}
\end{aligned}
$$

Reaction $\{1\}$ corresponds to the dissolution of ethyl acetate inserted into the reaction mass, and reaction $\{2\}$ is the chemical reaction itself.

\subsection{Mass balance}

In a semi-batch reactor, the instantaneous mass balance is written for an active chemical species $j$ :

$$
\frac{\mathrm{d} n_{j}^{\mathrm{E}}}{\mathrm{d} t}+V_{\mathrm{r}} \sum_{i=1}^{\mathrm{NR}} \nu_{i j} r_{i}=\frac{\mathrm{d} n_{j}}{\mathrm{~d} t}
$$

where $\left(\mathrm{d} n_{j}^{\mathrm{E}}\right) /(\mathrm{d} t)$ is the flow rate of introduction of $j, r_{i}$ the speed of the $i$ th reaction, with the stoichiometric coefficient $\nu_{i, j}$ and $V_{\mathrm{r}}$ the volume of the reaction mass.

The speed of $i$ th reaction is:

$r_{i}=k_{i} \prod_{j=1}^{\mathrm{NS}} C_{j}^{o_{i, j}}$

where $k_{i}$ is the kinetic constant, following Arrhenius' law (3), $o_{i, j}$ the order of constituent $j$ in the reaction $i$ and $C_{j}$ the concentration of constituent $j$ :

$C_{j}=\frac{n_{j}}{V_{\mathrm{r}}}$.

Let us define a new variable, a normalised extent for each reaction [16]:

$\chi_{i}=\frac{\left(\Delta n_{j}\right)_{i}}{\nu_{i, j} n_{0}} \quad \forall j$ such as $\nu_{i, j} \neq 0$

where $n_{0}$ is the reference mole number and $\left(\Delta n_{j}\right)_{i}$ the mole number of constituent $j$ which has reacted in the $i$ th reaction between initial and current times. Then the state of chemical species $j$ is given by its mole number:

$n_{j}=n_{j}^{\mathrm{E}}+n_{j, 0}+n_{0} \sum_{i=1}^{\mathrm{NR}} \nu_{i, j} \chi_{i}$ 
Table 1

Range of estimation

\begin{tabular}{lccllllll}
\hline Parameter & $k_{1}\left(\mathrm{~s}^{-1}\right)$ & $\Delta H_{1}$ & $\begin{array}{l}o_{1 \mathrm{~A}} \\
\left(\mathrm{~kJ} \mathrm{~mol}^{-1}\right)\end{array}$ & $\begin{array}{l}k_{2}\left(15^{\circ} \mathrm{C}\right) \\
\left(\mathrm{m}^{3} \mathrm{~mol}^{-1} \mathrm{~s}^{-1}\right)\end{array}$ & $\begin{array}{l}k_{2}\left(25^{\circ} \mathrm{C}\right) \\
\left(\mathrm{m}^{3} \mathrm{~mol}^{-1} \mathrm{~s}^{-1}\right)\end{array}$ & $\begin{array}{l}\Delta H_{2} \\
\left(\mathrm{~kJ} \mathrm{~mol}^{-1}\right)\end{array}$ & $\begin{array}{c}o_{2 \mathrm{~A}} \\
o_{2 \mathrm{~B}}\end{array}$ \\
\hline Minimum & $10^{-2}$ & 1 & 0.3 & $10^{-8}$ & $10^{-8}$ & -60 & 0.3 & 0.3 \\
Maximum & 1 & 10 & 2.5 & $10^{-3}$ & $10^{-3}$ & -40 & 2.5 & 2.5 \\
\hline
\end{tabular}

where $n_{j, 0}$ is the mole number of constituent $j$ at initial time. The differential of Eq. (15) leads to:

$$
\frac{\mathrm{d} n_{j}}{\mathrm{~d} t}=\frac{\mathrm{d} n_{j}^{\mathrm{E}}}{\mathrm{d} t}+0+n_{0} \sum_{i=1}^{\mathrm{NR}} \nu_{i j} \dot{\chi}_{i}
$$

The equality of (11) and (16) determines the production flow rate of the $i$ th reaction:

$n_{0} \dot{\chi}_{i}=r_{i} V_{\mathrm{r}}$

With the following definitions of dimensionless numbers:

$Y_{j, 0}=\frac{n_{j, 0}}{n_{0}}$

$Z_{j}=\frac{n_{j}^{\mathrm{E}}}{n_{0}}$

Eq. (12) becomes:

$\dot{\chi}_{i}=\frac{V_{\mathrm{r}}}{n_{0}}\left[k_{i}\left(\frac{n_{0}}{V_{\mathrm{r}}}\right)^{\sum_{i} o_{i, j}} \prod_{j=1}^{\mathrm{NS}}\left(Y_{j, 0}+Z_{j}+\sum_{i=1}^{\mathrm{NR}} \nu_{i, j} \chi_{i}\right)^{o_{i, j}}\right]$.

In our case, the number of chemical reaction is two. The system of differential Eq. (20) becomes:

$\dot{\chi}_{1}=k_{1}\left(\frac{n_{0}}{V_{\mathrm{r}}}\right)^{o_{1 \mathrm{~A}}-1}\left(Z_{\mathrm{A}}-\chi_{1}\right)^{o_{1 \mathrm{~A}}}$
$\dot{\chi}_{2}=k_{2}\left(\frac{n_{0}}{V_{\mathrm{r}}}\right)^{o_{2 \mathrm{~A}}+o_{2 \mathrm{~B}}-1}\left(\chi_{1}-\chi_{2}\right)^{o_{2 \mathrm{~A}}}\left(Y_{\mathrm{B} 0}-\chi_{2}\right)^{o_{2 \mathrm{~B}}}$

where $Z_{\mathrm{A}}$ is the introduction variable of ester evolving with time:

$Z_{\mathrm{A}}=\frac{n_{\mathrm{A}}^{\mathrm{E}}}{n_{0}}$

\subsection{Energy balance}

The thermal power released or absorbed by the chemical reactions is written as:

$q_{\mathrm{R}}^{\text {calc }}=-V_{\mathrm{r}} \sum_{i=1}^{\mathrm{NR}} r_{i} \Delta H_{i}$

Thanks to (17), (24) becomes:

$q_{\mathrm{R}}^{\text {calc }}=-n_{0} \sum_{i=1}^{\mathrm{NR}} \dot{\chi}_{i} \Delta H_{i}$

In our case, each phenomenon contributes to the total power according to its energy and kinetics:

$q_{\mathrm{R}}^{\text {calc }}=-n_{0}\left(\dot{\chi}_{1} \Delta H_{1}+\dot{\chi}_{2} \Delta H_{2}\right)$

The temperature is supposed to be controlled. But, as the volume is quite important (up to 21 ), the temperature cannot be considered to strictly follow the set temperature. Therefore, the experimental evolution of

Table 2

Parameters of the GA

\begin{tabular}{llllll}
\hline $\begin{array}{l}\text { Size of } \\
\text { population }\end{array}$ & $\begin{array}{l}\text { Selection } \\
\text { rate }\end{array}$ & $\begin{array}{l}\text { Mutation } \\
\text { rate }\end{array}$ & $\begin{array}{l}\text { Maximal number } \\
\text { of generations }\end{array}$ & $\begin{array}{l}\text { Characters per } \\
\text { parameter }\end{array}$ & $\begin{array}{l}\text { Positions of } \\
\text { cross-over }\end{array}$ \\
\hline 200 & 0.2 & $5 \%$ & 50 & 10 & after $1,3,6$ \\
\hline
\end{tabular}


Table 3

Parameters of Rosenbrock's method

\begin{tabular}{lllll}
\hline $\begin{array}{l}\text { Distance } \\
\text { between groups }\end{array}$ & $\begin{array}{l}\text { Limit group } \\
\text { number }\end{array}$ & $\begin{array}{l}\text { Precision } \\
\text { to stop }\end{array}$ & $\begin{array}{l}\text { Multiplication factor } \\
\text { for the constraints }\end{array}$ & $\begin{array}{l}\text { Maximum number } \\
\text { of iterations }\end{array}$ \\
\hline 0.1 & 3 & $10^{-5}$ & $10^{-4}$ & $10^{4}$ \\
\hline
\end{tabular}

the temperature must be taken into account in the expression of the kinetic constants. To solve our system composed of algebraic (23) and differential ((21) and (22)) equations, the Gear's method [17] was adopted: an algorithm based on the DASSL program [18] was written in $\mathrm{C}++$.

\subsection{Estimation of parameters}

\subsubsection{Range of search}

With the system of Eqs. (21)-(23) necessary to calculate the power released or absorbed by the reaction mass with Eq. (26), nine parameters must be estimated: the enthalpy of the two reactions $\left(\Delta H_{1}\right.$ and $\left.\Delta H_{2}\right)$, the two kinetic constants $\left(k_{1}\right.$ and $\left.k_{2}\right)$ which each have two parameters according to the temperature $\left(A_{i}\right.$ and $\left.E_{i}\right)$, and the three orders $\left(o_{1 \mathrm{~A}}, o_{2 \mathrm{~A}}\right.$ and $\left.o_{2 \mathrm{~B}}\right)$.

The chemical equation $\{1\}$ represents the mixing of the ester. As the range of temperature is quite narrow, the kinetic constant $k_{1}$ is supposed to be independent of temperature. So, there are only eight parameters to estimate.

The parameters are estimated in the range indicated in Table 1.
The units of kinetic constants $k_{1}$ and $k_{2}$ suppose that the orders are equal to one. The kinetic constants as well as the orders are estimated according to their logarithm. An additional constraint is added: the sum of the two enthalpies should be limited between -50 and $-40 \mathrm{~kJ} \mathrm{~mol}^{-1}$.

\subsubsection{Parameters of the estimation methods}

The parameters of the GA are given in Table 2.

The parameters of the Rosenbrock's method are given in Table 3.

The incertitude interval is calculated with a confidence level of $99 \%$. The amplitude of the interval for the parameter $\theta_{\mathrm{m}}$ [12] is:

$\operatorname{ampl}_{\mathrm{m}}=\sqrt{\operatorname{var} \theta_{\mathrm{m}}} t_{\beta / 2, s-n}$

where $t_{\beta / 2, s-n}$ is the Student's $t$-value for the $100(1$ $-\beta)$ confidence level and the number of data points $(s)$ minus the number of parameters to estimate $(n)$ degrees of freedom. The variance of the parameter $\theta_{\mathrm{m}}$ is:

$\operatorname{var} \theta_{\mathrm{m}}=\frac{2 f(\hat{\theta})}{s-n}\left(\frac{\partial^{2} f}{\partial \theta_{\mathrm{m}}^{2}}\right)_{\hat{\theta}}^{-1}$

Table 4

Results of the estimation before and after locally convergent method

\begin{tabular}{|c|c|c|c|c|c|c|}
\hline \multirow[t]{2}{*}{ Parameter } & \multicolumn{2}{|c|}{ 1st group (2675 iterations) } & \multicolumn{2}{|c|}{ 2nd group (2015 iterations) } & \multicolumn{2}{|c|}{ 3rd group (837 iterations) } \\
\hline & Before & After & Before & After & Before & After \\
\hline$k_{1}$ & 0.0139 & $0.0317 \pm 0.0006$ & 0.0139 & $0.0123 \pm 0.0006$ & 0.0109 & $0.0142 \pm 0.0002$ \\
\hline$\Delta H_{1}$ & 5910 & $6310 \pm 130$ & 5550 & $6400 \pm 150$ & 9510 & $7750 \pm 90$ \\
\hline$\Delta H_{2}$ & $-48,670$ & $-50,700 \pm 230$ & $-48,670$ & $-50,600 \pm 200$ & $-50,360$ & $-51,400 \pm 100$ \\
\hline$k_{2}\left(15^{\circ} \mathrm{C}\right)$ & $1.66 \times 10^{-5}$ & $1.32 \pm 0.02 \times 10^{-5}$ & $5.97 \times 10^{-6}$ & $1.51 \pm 0.03 \times 10^{-5}$ & $2.51 \times 10^{-5}$ & $2.5 \pm 0.2 \times 10^{-5}$ \\
\hline$k_{2}\left(25^{\circ} \mathrm{C}\right)$ & $4.49 \times 10^{-5}$ & $2.83 \pm 0.05 \times 10^{-5}$ & $2.01 \times 10^{-5}$ & $3.21 \pm 0.05 \times 10^{-5}$ & $8.76 \times 10^{-5}$ & $5.0 \pm 0.3 \times 10^{-5}$ \\
\hline$o_{1 \mathrm{~A}}$ & 1.015 & $1.178 \pm 0.008$ & 1.003 & $1.36 \pm 0.01$ & 1.024 & $1.38 \pm 0.01$ \\
\hline$o_{2 \mathrm{~A}}$ & 1.117 & $0.962 \pm 0.007$ & 1.383 & $0.945 \pm 0.007$ & 1.136 & $0.914 \pm 0.005$ \\
\hline$o_{2 \mathrm{~B}}$ & 0.958 & $0.977 \pm 0.005$ & 0.912 & $0.977 \pm 0.006$ & 0.912 & $0.939 \pm 0.005$ \\
\hline Criterion & $12,400.1$ & 3683.41 & $13,774.6$ & 3590.99 & $13,946.8$ & 4108.07 \\
\hline
\end{tabular}



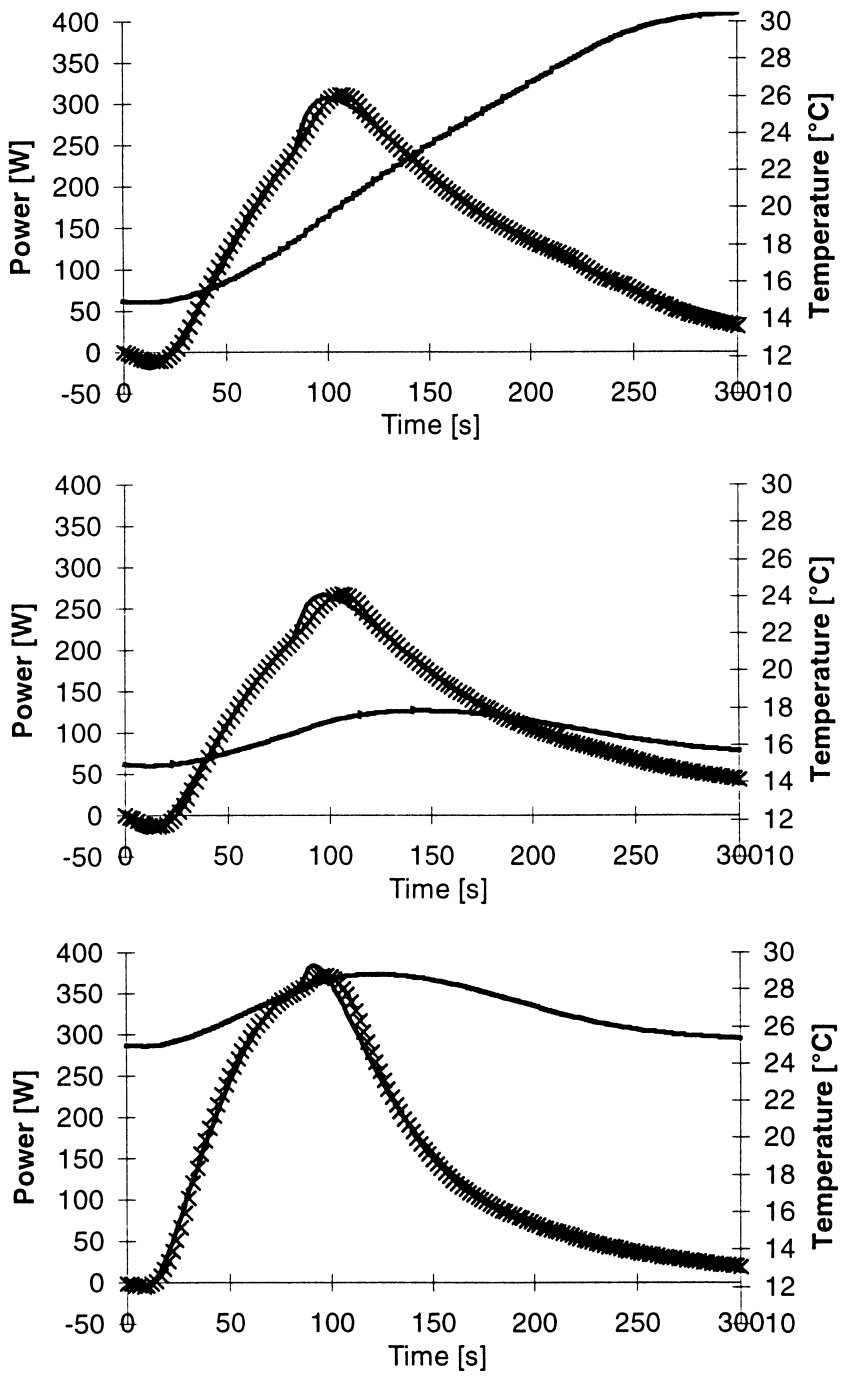

Fig. 7. Profiles of experimental and calculated power.

where $f(\hat{\theta})$ is the criterion at the estimated point and $\left(\left(\partial^{2} f\right) /\left(\partial \theta_{m}^{2}\right)\right)_{\hat{\theta}}^{-1}$ is the diagonal of the inverse of Hessian matrix.

\subsubsection{Results}

The first step, representing the GA, stops after the 18th generation, which has detected three groups. Starting from these three points, obtained thanks to the best individual of each of the three groups (given in the columns "Before" of Table 4), the local convergence method ends up at the results given in the columns "After' of Table 4. The units are indicated in Table 1.
By comparing the criterion, the second group seems to give the best results. Fig. 7 shows the good correlation between the three experiments and the

Table 5

Comparison of results with literature

\begin{tabular}{llll}
\hline & ICT [19] & Villermaux [16] & Present paper \\
& $C=0.01 \mathrm{~mol} \mathrm{l}^{-1}$ & $C=0.1 \mathrm{~mol} \mathrm{l}^{-1}$ & $C=1 \mathrm{~mol} \mathrm{l}^{-1}$ \\
\hline$k_{2}\left(15^{\circ} \mathrm{C}\right)$ & $5.45 \times 10^{-5}$ & $4.31 \times 10^{-5}$ & $1.51 \times 10^{-5}$ \\
$k_{2}\left(25^{\circ} \mathrm{C}\right)$ & $10.6 \times 10^{-5}$ & $7.78 \times 10^{-5}$ & $3.21 \times 10^{-5}$
\end{tabular}


Table 6

Results with the 20 best out of 100

\begin{tabular}{|c|c|c|c|c|c|c|c|c|c|c|}
\hline$\#$ & 1 & 2 & 3 & 4 & 5 & 6 & 7 & 8 & 9 & 10 \\
\hline Criterion before & 23,022 & 26,500 & 26,716 & 34,917 & 35,417 & 40,127 & 52,213 & 55,303 & 57,666 & 62,650 \\
\hline Number of iterations & 385 & 1690 & 1336 & 393 & 329 & 318 & 261 & 3307 & 299 & 734 \\
\hline Criterion after & 13,260 & 4160.1 & 4533.9 & 9597.1 & 34,378 & 6555.5 & 35,925 & 3728.1 & 44,515 & 13,886 \\
\hline$\#$ & 11 & 12 & 13 & 14 & 15 & 16 & 17 & 18 & 19 & 20 \\
\hline Criterion before & 62,771 & 68,023 & 71,747 & 77,501 & 83,653 & 84,241 & 84,848 & 85,859 & 86,680 & 87,359 \\
\hline Number of iterations & 220 & 380 & 1133 & 3560 & 5231 & 1197 & 911 & 285 & 2711 & 2198 \\
\hline Criterion after & 38,851 & 65,764 & 12,813 & 3679.0 & 3968.3 & 4519.3 & 5327.5 & 29,954 & 3704.3 & 3633.5 \\
\hline
\end{tabular}

model calculated with the values obtained by the second group.

\subsection{Discussion}

In literature, the kinetic experimental studies of ethyl acetate saponification are only carried out in aqueous reaction mass with low concentration. The International Critical Tables ([19], Vol. 7, pp. 129130) give a relation $\log _{10} k=f(T)$ in a range of temperature between $10^{\circ} \mathrm{C}$ and $45^{\circ} \mathrm{C}$ for concentration about $10^{-2} \mathrm{~mol} \mathrm{l}^{-1}$. Likewise, Villermaux [16], for temperatures of $26^{\circ} \mathrm{C}$ and $32^{\circ} \mathrm{C}$ and a concentration of $0.1 \mathrm{~mol}^{-1}$, gives the following values:

$A=3.05 \cdot 10^{3} \mathrm{~m}^{3} \mathrm{~mol}^{-1} \mathrm{~s}^{-1}$

$E=43,300 \mathrm{~J} \mathrm{~mol}^{-1}$

To compare the results, Table 5 gives the values of the kinetic constant at $15^{\circ} \mathrm{C}$ and $25^{\circ} \mathrm{C}$.

The kinetic constants calculated here are about three times as small as those of literature. However, the difference between the values of literature and those of the present study can be explained with the following points.

- The concentrations used here are 10 to 100 times higher than those of literature.
- The composition of the reaction mass is also quite different. This last point can be strengthened by the results obtained by Celdran et al. [20]. He shows in his works on the $n$-butyl acetate saponification that the proportion water/ethanol has a great influence on kinetic parameters.

- The orders calculated for the reactants are slightly different from 1 , unlike the studies in the literature where they are fixed to 1 .

For all these reasons, the comparison must be limited to some of idea of the value, which is respected. The experiments cannot be carried out with smaller concentrations because the signal measured would be too weak.

These results required 8447 estimations of the criterion, 5527 of them with the three local convergence methods, and 2920 with GA.

Furthermore, to test the efficiency of the GA, the same calculation is again carried out by bypassing the GA. A total of 100 points are taken in the parameter space, scattered on a uniform lattice (Sobol's type). The criterion is calculated for all the 100 points and only the 20 best ones are kept. These numbers are chosen arbitrarily. These 20 points serve to start a local convergence method. The value of the criterion before and after the estimation method, as well as the number of iterations are given in Table 6 .

Table 7

Values of the 20th point

\begin{tabular}{llllllll}
\hline$k_{1}$ & $\Delta H_{1}$ & $\Delta H_{2}$ & $k_{2}\left(15^{\circ} \mathrm{C}\right)$ & $k_{2}\left(25^{\circ} \mathrm{C}\right)$ & $o_{1 \mathrm{~A}}$ & $o_{2 \mathrm{~A}}$ & $o_{2 \mathrm{~A}}$ \\
\hline $0.0243 \pm 0.0007$ & $6190 \pm 130$ & $-50,700 \pm 100$ & $1.03 \pm 0.02 \times 10^{-5}$ & $2.22 \pm 0.04 \times 10^{-5}$ & $1.21 \pm 0.01$ & $0.96 \pm 0.01$ & $1.01 \pm 0.01$ \\
\hline
\end{tabular}


The 20th point gives the best result which is not so far from the one previously determined as indicated in Table 7. The units are indicated in Table 1.

But the total number of iterations is 26,978 , more than three times as high as with the hybrid method. It can also be noticed that the 14th and the 19th points give correct results. This fact points out the difficulty to determine the numbers of points initial and to be kept: if fewer points were kept, for instance only the 10 best out of the 100 initial points, the best results would not have been found. If more points are kept, this will increase the calculation time.

\section{Conclusion}

In this article, an estimation method for kinetic and energetic parameters is presented. The importance of changing parameters is underlined. This method considerably facilitates the search for the optimum.

The estimation method is a hybrid method, completing a GA with a local convergence method. The GA detects the most relevant zones in the parameter space, then the local convergence method rapidly determines the relative minimum of the zone, and so on for all the zones detected. So the method is able to give not just a single answer, but a set of answers. Thus the user decides, according to the physical meaning of the solutions, which one is the most probable answer to the problem.

This method is applied to a real complex case: the determination of kinetic and energetic parameters of the saponification of ethyl acetate in hydroalcoholic reaction mass with a concentration of $1 \mathrm{~mol} \mathrm{l}^{-1}$. The values of the parameters estimated allow to represent all the three experiments. The hybrid estimation method is compared to a much more simple one, where the 20 best points out of 100 scattered in the whole parameter space serve to initiate a local convergence method. This second method is dependent of the initial number of points and the number of points to keep. Indeed, to avoid missing the physically significant solution, the numbers of points need to be high. But this will extensively increase the calculation time. So this method is costly in time in comparison to the hybrid method. The latter allows to determine more surely and more rapidly the solution to the problem.

\section{References}

[1] L. Balland, Contribution à l'Estimation des Paramètres Cinétiques et Énergétiques de Systèmes Chimiques en milieu Homogène et Hétérogène dans un Réacteur Calorimétrique. Thesis of the University of Rouen, France, 1997, 245 pp.

[2] D.B. Hibbert, Genetic algorithms in chemistry, Chemometrics and Intelligent Laboratory Systems 19 (1993) 277-293.

[3] L. Balland, J.-M. Cosmao, N. Mouhab et al., Estimation des Paramètres de Modèles Cinétiques par Mesure des Flux d'Énergie dans un Réacteur Double Enveloppe, Entropie 186 (1994) 25-32.

[4] J.M. Sobol, On the systematic search in a hypercube, SIAM J. Number Anal. 16 (5) (1979) 790-793.

[5] F. Bicking, C. Fonteix, J.-P. Corriou, I. Marc, Global optimisation by artificial life: a new technique using genetic population evolution, Operations Research 28 (1) (1994) 23-36.

[6] D.B. Hibbert, A hybrid genetic algorithm for the estimation of kinetic parameters, Chemometrics and Intelligent Laboratory Systems 19 (1993) 319-329.

[7] H.H. Rosenbrock, An automatic method for finding the greatest or the least value of a function, Comput. J. 3 (1960) 175-184.

[8] N.H. Chen, Determination of Arrhenius constants by linear and non linear fitting, AIChE J. 38 (4) (1992) 626-628.

[9] P. Billardello, X. Joulia, J.M. Le Lann et al., A general strategy for parameter estimation in DAE Systems, Comput. Chem. Eng. 17 (5-6) (1993) 517-525.

[10] D.J. Pritchard, D.W. Bacon, Prospect for reducing correlation among parameter estimates in kinetic models, Chem. Eng. Sci. 33 (1978) 1539-1543.

[11] Ph. Swaels, Modélisation et Simulation Dynamique de Réacteurs Chimiques Discontinus. Thesis of the University of Rouen, France, 1995, 304 pp.

[12] P.J. Pigram, R.N. Lamb, D.B. Hibbert, R.E. Collins, Modeling of the desorption behavior of microporous amorphous hydrogenated carbon films, Langmuir 10 (1994) 142-147.

[13] A.O. Griewangk, Generalized descent for global optimisation, J.O.T.A. 34 (1981) 11-39.

[14] H.P. Schwefel, Nümerische Optimierung von ComputerModellen mittels der Evolutionsstrategie. Interdisciplinary System Research, Birkhäuser, Basel, 1977, p. 26.

[15] L. Balland, N. Mouhab, S. Alexandrova, J.-M. Cosmao, L. Estel, Determination of kinetic and energetic parameters of chemical reactions in a heterogeneous liquid/liquid system, Chem. Eng. Technol. 22 (4) (1999) 321-329.

[16] J. Villermaux, Génie de la Réaction Chimique, Conception et fonctionnement des Réacteurs, Technique et Documentation Lavoisier, Paris, 1993, 448 pp.

[17] C.W. Gear, The automatic integration of ODE, Numerical Mathematics 14 (3) (1971) 176-179. 
[18] L. Petzold, Differential/algebraic equations are not ODE's, SIAM J. Sci. Stat. Comput. 3 (3) (1982) 367-384.

[19] E.W. Washburn (Ed.), International Critical Tables of Numerical Data, Physics, Chemistry and Technology, Vol. 7, McGraw-Hill, New York, pp. 1926-1933.
[20] E.W. Celdran, M.V. Ramon, P. Martinez, Influence of the polarity of the medium on the alkaline hydrolysis of $n$-butyl acetate in hydroalcoolic mixtures, Z. Naturforsch. 32a (1977) 1496-1500. 